Editorial

\title{
Alineación en PTR: Una controversia en evolución aun no resuelta
}

\section{Alignment in TKA: An Unresolved Controversy}

\author{
Rafael Calvo Rodríguez ${ }^{1}$ Javier González Almonacid ${ }^{1}$ \\ ${ }^{1}$ Clínica Alemana - Universidad del Desarrollo \\ Rev Chil Ortop Traumatol 2021;62(2):e75-e76.
}

El problema

A los 15 años la supervivencia de estos implantes es superior al $96 \%$ y a los 25 años superan el $82 \%^{1}$ no obstante, esta buena supervivencia se oscurece por un porcentaje elevado de pacientes, alrededor del $20 \%$, que continúan con dolor y otras molestias inespecíficas en la rodilla con el paso del tiempo. ${ }^{2}$

La insatisfacción luego de una Artroplastía Total de Rodilla (ATR) es un fenómeno bastante documentado en la literatura. ${ }^{3}$

Sobre la base de una revisión del Registro Sueco, Dunbar et al informó que el $17 \%$ de los pacientes no estaban satisfechos con el resultado de la ATR. ${ }^{4}$ Baker et al. revaluaron los datos del Registro Nacional de Inglaterra y Gales: el 71\% de los pacientes refirió una mejoría de los síntomas de la rodilla, pero sólo el $22 \%$ calificaron los resultados como "excelente" 5 .

En la búsqueda de soluciones para mejorar estos resultados, se han planteado nuevas tecnologías de ayuda para el implante (desde la navegación hasta la robótica) $\mathrm{Si}$ bien esto es un problema multifactorial, en el último tiempo se ha puesto énfasis en el alineamiento como una causa modificable de este problema. ${ }^{6}$

Existe controversia respecto al tipo de alineación más adecuada en ATR. ${ }^{6}$

El concepto de rodilla balanceada no tiene una definición clara y puede conseguirse de más de una manera. Existen 2 técnicas clásicas descritas para esto: La resección medida (measured resection) y el balance de la separación de fémur y tibia (gap balancing). ${ }^{7}$

En la resección medida (MR) se usan las referencias anatómicas para colocar el implante. Los cortes óseos son realizados independientemente de la situación ligamentosa y se basan en el eje transepicodíleo, el eje anteroposterior femoral o eje de Whiteside y el eje posterior de los cóndilos. El cirujano sigue las guías del instrumental, intentando que las resecciones óseas femoral distal y posterior sean similares; los ligamentos se adaptan después a través de liberaciones de partes blandas.

En la técnica de gap balancing (TG) se usan sistemas de distracción para definir la mejor posición de la artroplastia y posteriormente se adaptan a ello los cortes óseos y las liberaciones ligamentosas.

Pero independiente de la técnica que se utilice, el objetivo por décadas ha sido el mismo, obtener una rodilla mecánicamente alineada. ${ }^{8}$

Lo clásico sigue siendo alineación mecánica pero este enfoque ignora la anatomía de la articulación nativa y la relación entre el origen e inserción de los tejidos blandos. Lo nuevo: Alineación cinemática, funcional, anatómica etc.

El alineamiento mecánico busca una rodilla que respete el eje mecánico de la extremidad inferior, en que los ejes mecánicos de fémur y tibia formen un ángulo de $180^{\circ}$ entre ellos. ${ }^{8}$ El problema es que este eje es nativamente neutro en solo un determinado porcentaje de la población, por lo que ha sido puesto en duda como parámetro a seguir. Se ha descrito varo constitucional en hasta un 30\% de los hombres y $17 \%$ de las mujeres. ${ }^{9}$

No se puede dudar sobre los excelentes resultados que se han logrado con la ATR alineada mecánicamente. ${ }^{8}$ Pero nuevos estudios han generado mayor interés en un nuevo concepto de alineación; la alineación cinemática. Dos ensayos clínicos randomizados y otros estudios multicéntricos mostraron que pacientes tratados con alineación cinemática reportaron una diferencia significativamente mejor respecto a alineamiento mecánico en alivio del dolor, función, flexión y sensación más normal de la rodilla, con una sobrevida del implante similar a 2, 3 y 6 años. ${ }^{10-12}$

La alineación cinemática busca reconstruir el eje de la extremidad previo al implante de la Artroplastía (eje pre

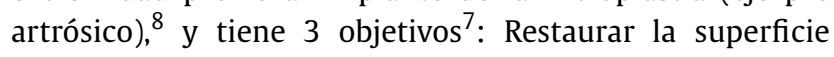
articular tibiofemoral nativa, Restaurar el alineamiento

\author{
Address for correspondence Rafael DOI https://doi.org/ \\ Calvo, MD, Clínica Alemana de \\ Santiago SA, Vitacura, Región \\ Metropolitana, Chile \\ (e-mail: rcalvo61@gmail.com). \\ 10.1055/s-0041-1735514. \\ ISSN $0716-4548$.
}

(c) 2021. Sociedad Chilena de Ortopedia y Traumatologia. All rights reserved.

This is an open access article published by Thieme under the terms of the Creative Commons Attribution-NonDerivative-NonCommercial-License, permitting copying and reproduction so long as the original work is given appropriate credit. Contents may not be used for commercial purposes, or adapted, remixed, transformed or built upon. (https://creativecommons.org/ licenses/by-nc-nd/4.0/)

Thieme Revinter Publicações Ltda., Rua do Matoso 170, Rio de Janeiro, RJ, CEP 20270-135, Brazil 
nativo de la extremidad inferior y restaurar la laxitud nativa de la rodilla.

Riviere et al definió 5 métodos de alineamiento del implante ${ }^{13}$ : Mecánico, mecánico ajustado, anatómico, cinemático y cinemático restringido. Recientemente Oussedick et al definió además el alineamiento funcional. ${ }^{14}$ En su estudio plantea que el alineamiento mecánico ignora la importancia de las partes blandas. El alineamiento cinemático, por su parte, respeta las partes blandas, pero ignora el entorno mecánico, por lo que propone al alineamiento funcional como una técnica híbrida que respeta la tensión de partes blandas, pero al mismo tiempo el entorno mecánico de la extremidad.

A nuestro entender es en esta línea donde se planteará el mayor desarrollo y evolución de la alineación en prótesis de rodilla.

La ATR con alineamiento funcional muestra un camino para lograr una cinemática paciente específica, manipulando las resecciones óseas y modificando levemente la posición de los componentes para limitar la necesidad de liberación de tejidos blandos periarticulares. ${ }^{15}$ Esto se logra con asistencias técnicas avanzadas, como cirugía robótica o navegación. La precisión adicional lograda con esas técnicas permite que un alineamiento de extremidades inferiores no neutro pueda lograrse de manera más reproducible. ${ }^{16}$ Con el alineamiento funcional mediante cirugía robótica, los gaps pueden balancearse cambiando la dirección de los componentes en los 3 planos. Este posicionamiento puede ser individualizado a la rodilla del paciente, manteniendo límites seguros de alineamiento, lo cual considera un rango seguro de $0 \pm 3^{\circ}$ de alineamiento coronal. ${ }^{14}$

Este modelo híbrido que provee una rodilla balanceada pero que se mantiene dentro de límites de seguridad del alineamiento mecánico podría tener resultados promisorios, ${ }^{14}$ disminuyendo la morbilidad asociada a la gran liberación de partes blandas que a veces requiere el alineamiento mecánico y pudiendo lograr una mejor satisfacción de los pacientes sin comprometer la longevidad del implante. ${ }^{15}$

La literatura publicada hasta ahora que compara alineamiento mecánico versus alineación cinemática muestra resultado similares a corto plazo, con algunos estudios describiendo una diferencia positiva para la alineación cinemática. $^{8}$ Es posible que la alineación funcional sea el método que lleve a mejores resultados pero aún la literatura es escasa por lo que el objetivo óptimo de alineación sigue siendo una pregunta abierta cuya respuesta deberá seguir investigándose.

Conflict of Interest

None declared

\section{Bibliografia}

1 National Joint Registry. 16th Annual report. 2019https://reports. njrcentre.ORG.UK/PORTALS/0/PDFDOWNLOADS/NJR\%2016TH\% 20ANNUAL\%20REPORT\%202019.PDF Date accessed 22 Jan 2020

2 Baker PN, van der Meulen JH, Lewsey J, Gregg PJNational Joint Registry for England and Wales Data from the National Joint Registry for England and Wales. The role of pain and function in determining patient satisfaction after total knee replacement. J Bone Joint Surg Br 2007;89(07):893-900

3 Nam D, Nunley RM, Barrack RL. Patient dissatisfaction following total knee replacement: a growing concern? Bone Joint J 2014;96B(11, Supple A) $96-100$

4 Dunbar MJ, Richardson G, Robertsson O. I can't get no satisfaction after my total knee replacement: rhymes and reasons. Bone Joint J 2013;95-B(11, Suppl A)148-152

5 Baker PN, Rushton S, Jameson SS, Reed M, Gregg P, Deehan DJ. Patient satisfaction with total knee replacement cannot be predicted from pre-operative variables alone: A cohort study from the National Joint Registry for England and Wales. Bone Joint J 2013;95-B(10):1359-1365

6 Clement ND, Deehan DJ. Minimum reporting criteria for robotic assisted total knee arthroplasty studies. Alignment and balancing techniques should both be defined. Bone Joint Res. 2020;9:279-81

7 Howell SM. Kinematically Aligned Total Knee Arthroplasty. En Insall and Scott Surgery of the Knee. 6th edition New York: Elsevier; 2018:1784-1796

8 The alignment of the knee replacement. Old myths and new controversies. Rev Esp Cir Ortop Traumatol (Engl Ed). 2021 Apr 22; S18888-4415(21)52-7

9 Bellemans J, Colyn W, Vandenneucker H, Victor J. The Chitranjan Ranawat award: Is neutral mechanical alignment normal for all patients? The concept of constitutional varus. Clin Orthop Relat Res. 2012;470:45-53

10 Calliess T, Bauer K, Stukenborg-Colsman C, et al. PSI kinematic versusnon-PSI mechanical alignment in total knee arthroplasty: a prospective, randomized study. Knee Surg Sports Traumatol Arthrosc 2016;27:1-6

11 Dossett HG, Estrada NA, Swartz GJ, LeFevre GW, Kwasman BG. A randomised controlled trial of kinematically and mechanically aligned total knee replacements: two-year clinical results. Bone Joint J 2014;96-B(07):907-913

12 Howell SM, Papadopoulos S, Kuznik K, Ghaly LR, Hull ML. Does varus alignment adversely affect implant survival and function six years after kinematically aligned total knee arthroplasty? Int Orthop 2015;39(11):2117-2124

13 Rivière C, Iranpour F, Auvinet E, et al. Alignment options for total knee arthroplasty: A systematic review. Orthop Traumatol Surg Res 2017;103(07):1047-1056

14 Oussedik S, Abdel MP, Victor J, Pagnano MW, Haddad FS. Alignment in total knee arthroplasty. Bone Joint J 2020;102-B (03):276-279

15 Kayani B, Konan S, Tahmassebi J, Oussedik S, Moriarty PD, Haddad FS. A prospective double-blinded randomised control trial comparing robotic arm-assisted functionally aligned total knee arthroplasty versus robotic arm-assisted mechanically aligned total knee arthroplasty. Trials 2020;21(01):194

16 Kayani B, Konan S, Pietrzak JRT, Huq SS, Tahmassebi J, Haddad FS. The learning curve associated with robotic-arm assisted unicompartmental knee arthroplasty: a prospective cohort study. Bone Joint J 2018;100-B(08):1033-1042 